\title{
Déclaration sur la strongyloïdose disséminée : lignes directrices en matière de prévention, d'évaluation et de prise en charge du CCMTMV
}

\author{
Boggild $\mathrm{AK}^{1,2,3}$, Libman $\mathrm{M}^{4}$, Greenaway $\mathrm{C}^{5}$, McCarthy $\mathrm{AE}^{6}$, au nom du Comité consultatif de la \\ médecine tropicale et de la médecine des voyages (CCMTMV)*
}

\section{Résumé}

Contexte : Strongyloides stercoralis est un nématode parasite que l'on trouve chez l'homme, avec une prévalence plus élevée dans les régions tropicales et subtropicales partout dans le monde. Si elle n'est pas traitée, l'infection peut évoluer en strongylö̈dose disséminée, maladie grave qui peut être fatale.

Objectif : Fournir des conseils cliniques sur la prévention, l'évaluation et la prise en charge de la strongyloïdose disséminée.

Méthodologie : Une revue de la littérature a été réalisée pour évaluer les données probantes actuelles et identifier tous les examens systématiques, les rapports de cas, les lignes directrices et les littératures médicales revues ou non revues par les pairs. Le Comité consultatif de la médecine tropicale et de la médecine des voyages (CCMTMV) a constitué un groupe de travail pour élaborer cette déclaration, qui a ensuite été examinée d'un œil critique et approuvée par tous les membres du CCMTMV.

Recommandations : Le CCMTMV recommande que le dépistage de la strongyloïdose soit envisagé pour les personnes sujettes à un risque épidémiologique ou de comorbidités qui les exposent à un risque d'hyperinfection et de dissémination du Strongyloides. Les personnes exposées au risque le plus élevé d'hyperinfection et de dissémination sont celles qui sont nées dans une région où le Strongyloides est endémique, qui présentent une immunosuppression iatrogène ou une infection par le virus T-lymphotrope humain intercurrent (HTLV-1). Le diagnostic de la strongyloïdose est fondé sur des tests sérologiques ou l'examen des selles et autres échantillons cliniques pour la recherche de larves. Pour les cas présumés et confirmés, il est conseillé de s'adresser à un spécialiste en médecine tropicale ayant une expertise dans la prise en charge de la strongylö̈dose. Un algorithme de diagnostic et de traitement de la strongyloïdose a été élaboré en tant qu'outil de référence.

Conclusion : La strongyloïdose est relativement répandue dans la population migrante mondiale et le dépistage de la maladie doit être fondé sur une évaluation des risques individuels. Un outil pratique dont le clinicien pourra se servir pour la prévention, l'évaluation et la prise en charge de la strongyloïdose disséminée au Canada est désormais disponible.
Rattachement des auteurs

'Unité des maladies tropicales, Hôpital général de Toronto, Réseau universitaire de santé, Toronto (Ontario)

2Département de médecine, Université de Toronto, Toronto (Ontario)

${ }^{3}$ Laboratoires de santé publique Ontario, Toronto (Ontario)

${ }^{4}$ Centre de médecine tropicale J. D. MacLean, Division des maladies infectieuses, Département de microbiologie, Centre universitaire de santé McGill, Montréal (Québec)

${ }^{5}$ Hôpital général juif, Division des maladies infectieuses, Centre d'épidémiologie clinique, Lady Davis Institute for Medical Research, Université McGill, Montréal (Québec)

${ }^{6} \mathrm{Clinique}$ de médecine tropicale et de santé internationale, Division des maladies infectieuses, Campus général de l'Hôpital d'Ottawa, Ottawa (Ontario)

\section{*Correspondance}

catmat.secretariat@phac-aspc. gc.ca

Citation proposée : Boggild AK, Libman M, Greenaway C, McCarthy AE, au nom du Comité consultatif de la médecine tropicale et de la médecine des voyages (CCMTMV). Déclaration sur la strongyloïdose disséminée lignes directrices en matière de prévention, d'évaluation et de prise en charge du CCMTMV. Relevé des maladies transmissibles au Canada 2016;42:14-22. https://doi.org/10.14745/ccdr.v42i01a03f

\section{Préambule}

Le Comité consultatif de la médecine tropicale et de la médecine des voyages (CCMTMV) donne de façon continue à l'Agence de la santé publique du Canada des conseils opportuns de nature médicale, scientifique et sanitaire concernant les maladies infectieuses tropicales et les risques pour la santé associés aux voyages internationaux. L'Agence reconnait que les recommandations et les conseils formulés dans cette déclaration reposent sur les pratiques médicales et les connaissances scientifiques les plus récentes et les diffuse dans le but d'informer les voyageurs ainsi que les professionnels de la santé qui sont appelés à leur prodiguer des soins.

Les personnes qui administrent ou utilisent des médicaments, des vaccins ou d'autres produits devraient bien connaître la monographie des produits, ainsi que toute autre norme ou instruction approuvée concernant leur usage. Les recommandations relatives à l'usage des produits et les autres renseignements présentés ici 
peuvent différer de ceux qui figurent dans la monographie ou toute autre norme ou instruction approuvée semblable établie par les fabricants autorisés. Les fabricants font approuver leurs produits et démontrent l'innocuité et l'efficacité de ceux-ci uniquement lorsque ces produits sont utilisés conformément à la monographie ou à toute autre norme ou instruction approuvée semblable.

\section{Introduction}

La strongyloïdose est une maladie causée par un nématode (ver rond) qui est principalement présent dans les régions tropicales et subtropicales, mais également dans les régions au climat tempéré. II n'y a aucune donnée précise sur la prévalence dans les pays endémiques, mais l'on estime que 30 à 100 millions de personnes sont infectées dans le monde (1). La plupart des personnes infectées par le Strongyloides sont asymptomatiques et ne sont pas conscientes de leur infection. Néanmoins, les personnes qui sont immunodéprimées courent un risque de développer une forme sévère de strongyloïdose disséminée qui, si elle n'est pas traitée, peut entraîner une maladie potentiellement mortelle (2). Bien que la strongylö̈dose soit traditionnellement considérée comme une maladie tropicale, l'immigration et l'augmentation des voyages internationaux sont à l'origine d'une augmentation des cas nécessitant des soins médicaux au Canada.

Les objectifs de cette déclaration sont les suivants :

- Accroître la sensibilisation à la strongyloïdose disséminée auprès des cliniciens susceptibles de rencontrer ces cas (notamment les cliniciens de première ligne tels que les urgentologues, les spécialistes des maladies infectieuses, les rhumatologues, les dermatologues, les gastroentérologues, les oncologues, les intensivistes et les équipes de transplantation).

- Aider les cliniciens à prévenir, évaluer et prendre en charge la strongyloïdose disséminée.

\section{Méthodologie}

La présente déclaration a été créée après que le CCMTMV a identifié la nécessité d'informer les cliniciens canadiens au sujet de la strongyloïdose disséminée. Un groupe de travail du CCMTMV a été constitué et un membre a été élu pour diriger l'élaboration de la déclaration. La littérature disponible a été évaluée pour identifier les examens systématiques, les rapports de cas, les lignes directrices et les littératures médicales revues ou non revues par les pairs. S'appuyant sur les données probantes recueillies et les opinions d'experts, un algorithme de diagnostic et de traitement de la strongyloïdose a été conçu en tant qu'outil de référence pour les cliniciens au Canada. La déclaration a ensuite été examinée d'un œil critique et approuvée par tous les membres du CCMTMV.

\section{Épidémiologie}

Strongyloides stercoralis est un nématode parasite que I'on trouve chez l'homme, dans les régions tropicales et subtropicales du monde entier. La prévalence de l'infection est élevée dans les Caraïbes, en Afrique de l'Est et de l'Ouest et particulièrement en Asie du Sud-Est (3). Les données montrent que 10 à $40 \%$ de la population des régions tropicales et subtropicales est affectée par la strongyloïdose, avec des taux atteignant $60 \%$ dans les pays où la situation écologique et socioéconomique est favorable à la transmission de $S$. stercoralis (3). Une étude canadienne sur les réfugiés a mis en évidence une séroprévalence de $77 \%$ parmi les réfugiés provenant du Cambodge et une séroprévalence de $12 \%$ parmi les réfugiés du Vietnam (4). Par ailleurs, la strongyloïdose était le cinquième diagnostic le plus courant parmi les 1321 nouveaux immigrants malades ayant sollicité des soins auprès d'un établissement du Canadian Travel Medicine Network (CanTravNet) réseau canadien de la médecine des voyages sur une période de trois ans $(5,6)$. Étant donné que 6,8 millions de Canadiens sont nés à l'étranger, dont environ $85 \%$ dans des régions où la strongyloïdose est endémique (7), une proportion substantielle des immigrants et des réfugiés au Canada présente un risque de développer la strongyloïdose. L'Asie demeure la principale région d'origine des immigrants au Canada, et les Philippines, la Chine et l'Inde sont les principaux pays d'origine (7). Les populations d'immigrants en provenance d'Afrique, des Caraïbes, d'Amérique centrale et du Sud augmentent également au fil du temps (7).

Au Canada, le nombre de personnes atteintes de strongyloïdose intestinale simple est estimé à environ 2,5 millions, avec une prévalence supposée de $40 \%$ (3) pour les personnes provenant des pays d'origine de cette infection. Cette estimation exclut les cas de strongyloïdose contractée lors d'un voyage, qui devraient représenter une minorité des cas au Canada. Cependant, il est important de reconnaître que même les séjours de courte durée dans des régions fortement endémiques peuvent être associés à l'acquisition de la strongyloïdose $(8,9,10)$.

Il est difficile d'estimer la proportion de réfugiés et d'immigrants canadiens susceptibles de développer une strongyloïdose disséminée, comme les personnes qui présentent une immunosuppression iatrogène ou une co-infection par le virus T-lymphotrope humain intercurrent (HTLV-1).

\section{Pathogenèse}

La strongyloïdose est acquise lorsque des larves infectieuses, présentes dans le sable ou la terre, pénètrent une peau humaine intacte, et, après une phase de migration obligatoire dans les tissus, se développent jusqu'au stade adulte dans l'intestin grêle. Contrairement à d'autres helminthes parasitaires, le Strongyloides a une durée de vie indéfinie chez l'hôte humain. En raison d'un cycle d'auto-infection lors duquel les larves au stade infectieux pénètrent à nouveau dans la peau ou l'intestin de l'hôte, la maladie clinique est un risque à vie si aucun traitement n'est dispensé. 


\section{Tableau clinique}

L'infection par Strongyloides peut provoquer un spectre de maladie allant de l'éosinophilie asymptomatique aux symptômes gastro-intestinaux jusqu'à une auto-infection accélérée (ou "syndrome d'hyperinfection ") ou encore une maladie disséminée fulminante et fatale. L'immunosuppression telle que celle qui se produit dans le cadre d'une corticothérapie prolongée, d'une infection par le HTLV-1 ou d'une hémopathie maligne, est un facteur de risque pour la strongyloïdose disséminée $(11,12,13,14)$, entité dont la documentation existante révèle qu'elle est associée à un taux de mortalité supérieur à $85 \%(15,16)$. Les mécanismes exacts pour le contrôle immunologique de cette infection ne sont pas clairs.

\section{Diagnostic et dépistage}

Le consortium canadien sur la santé des immigrants et des réfugiés $(\mathrm{CCRIH})$ a récemment recommandé de procéder au dépistage du Strongyloides uniquement pour les réfugiés provenant d'Asie du Sud-Est et d'Afrique subsaharienne (17). Un dépistage plus large n'a pas été recommandé car il y a peu de données disponibles sur la prévalence de la strongyloïdose dans les populations immigrantes et le dépistage sérologique n'est pas facilement ou rapidement disponible dans de nombreuses régions du Canada. Notre expérience clinique collective nous a toutefois montré que la strongyloïdose est largement répandue au sein de la population migrante mondiale. Le dépistage doit donc s'appuyer sur une évaluation des risques, en tenant compte du risque d'exposition au Strongyloides, du risque de maladie disséminée et de la présence d'un syndrome clinique (y compris pour les personnes asymptomatiques qui doivent subir une immunosuppression iatrogène). Ce constat est étayé par une série de dix cas documentés de strongylö̈dose disséminée recensés sur une période de sept mois à Toronto. Tous ces cas concernaient des immigrants au Canada, en provenance d'Asie du Sud-Est, des Caraïbes, d'Amérique du Sud ou d'Italie (11). Collectivement, les membres du CCMTMV ont contribué à la prise en charge de patients atteints de strongyloïdose ayant voyagé ou séjourné en Méditerranée, dans toutes les régions d'Afrique, aux Caraïbes, en Amérique latine, en Asie du Sud, dans le sous-continent indien et dans la région à très haut risque d'Asie du Sud-Est. Ainsi, nous recommandons un examen attentif des risques épidémiologiques, comme il est indiqué ci-après, pour une prise de décision éclairée en matière de dépistage.

En raison de la faible sensibilité de l'examen des selles pour rechercher des œufs et des parasites, due à la faible charge larvaire et à une excrétion intermittente dans les selles, les tests sérologiques constituent la méthode diagnostique préférée pour les patients chez qui une strongyloïdose intestinale simple est suspectée.

Il est important de noter que la sensibilité de la sérologie peut être réduite chez les patients immunodéprimés, en particulier en raison d'une infection par le HTLV-1, d'une hématopathie maligne et à la chimiothérapie associée $(18,19)$. Ces personnes risquent également de développer une strongyloïdose disséminée. De manière générale, le dépistage doit donc comporter des tests sérologiques et un examen des selles, comme il est indiqué ci-après. L'examen parasitologique des selles qui s'avère positif pour les larves de Strongyloides doit conduire au dépistage de l'infection par le HTLV-1 et à la consultation d'un spécialiste en médecine tropicale avec une expertise dans la prise en charge de la strongyloïdose. Les médecins membres du Réseau canadien sur le paludisme sont disponibles pour fournir des conseils dans de pareils cas (20).

\section{Traitement}

Le médicament de choix pour le traitement de la strongyloïdose intestinale et asymptomatique simple est l'ivermectine $(15,21)$ administrée en deux doses. Les personnes nées ou ayant résidé de manière prolongée dans des zones de forêt pluviale d'Afrique centrale (p. ex. au Cameroun, en Guinée équatoriale, au Gabon, en République centrafricaine, au Congo et en République démocratique du Congo, ainsi que les régions du sud du Nigeria, du Tchad, du Sud-Soudan et le nord de l'Angola) doivent avoir une microfilarémie élevée (loase) exclue avant l'administration de l'ivermectine. Pour cela, un examen des frottis sanguins doit être réalisé en journée pour rechercher des microfilaires de Loa loa.

Pour l'hyperinfection par Strongyloides ou le syndrome de dissémination, le CCMTMV recommande un double traitement par ivermectine et albendazole, comme il est décrit ci-après, étayé par les données de déclaration de cas $(11,22,23,24,25)$, des opinions d'experts et de l'expérience clinique des membres du CCMTMV. Les échantillons cliniques, y compris les expectorations et les selles, doivent être régulièrement contrôlés au cours du traitement de la dissémination ou de l'hyperinfection par Strongyloides pour s'assurer de l'élimination des larves.

Afin de prévenir le développement de la strongyloïdose disséminée, les patients présentant un risque de complications ou d'échec du traitement, comme ceux atteints d'une co-infection par le HTLV-1 ou Loa loa, doivent être dirigés vers un spécialiste de la médecine tropicale avec une expertise dans la prise en charge de ces infections. II n'y a aucune preuve pour étayer le fait qu'une stratégie de dépistage et de traitement soit supérieure ou plus efficace qu'une administration empirique d'ivermectine pour les personnes à risque devant subir une suppression immunitaire (26). Comme l'accès à l'ivermectine est limité au Canada, le CCMTMV recommande que le traitement empirique soit réservé aux personnes dont la suppression immunitaire prévue ne peut attendre le dépistage, comme il est décrit à l'étape 3 de l'algorithme de diagnostic et de traitement ci-après.

Tout patient ayant une strongyloïdose disséminée doit également recevoir un traitement empirique avec des antibiotiques à large spectre afin de couvrir la septicémie polymicrobienne, qui est une complication fréquente du syndrome d'hyperinfection. L'albendazole et l'ivermectine sont des agents de grossesse de catégorie C. Chez une personne enceinte atteinte d'une dissémination ou d'une hyperinfection par Strongyloides, les avantages du traitement surpassent vraisemblablement les risques liés à la nature mortelle de la strongyloïdose disséminée. L'ivermectine et l'albendazole sont uniquement disponibles au Canada par l'intermédiaire du Programme d'accès spécial de Santé Canada (27). Le délai de traitement des demandes soumises au programme est 
généralement d'une semaine. Les demandes urgentes, pour le jour même, peuvent toutefois être effectuées par téléphone.

\section{Questions de prévention des infections}

Les patients atteints de strongyloïdose disséminée doivent être pris en charge avec toutes les précautions contre la transmission par contact, en raison du risque de présence de larves infectieuses filariformes dans les effluents tels que les selles, I'urine, les expectorations et les aspirats endotrachéaux. La plupart de ces patients sont gravement malades et nécessitent des soins médicaux et des soins infirmiers intensifs. Ainsi, il est essentiel de prendre toutes les précautions pour prévenir la transmission nosocomiale aux travailleurs de la santé. II faut toutefois noter que la transmission nosocomiale est un risque théorique qui n'a pas été bien documenté dans la littérature $(28,29)$.

Il est également recommandé au personnel de laboratoires de prendre toutes les précautions contre la transmission par contact, en raison du risque d'exposition à des larves infectieuses filariformes, notamment dans les cultures de selles ou d'expectorations envoyées au laboratoire pour exclure une infection bactérienne. Les plaques de gélose des prélèvements provenant de patients atteints de strongyloïdose disséminée doivent être manipulées avec des gants et scellées avec du ruban Parafilm ${ }^{\circledR}$. Les larves filariformes des autres helminthes nématodes sont sensibles à l'éthanol à $70 \%$ pendant 10 minutes, au Dettol ${ }^{\circledR}$ à $0,5 \%$ pendant 20 minutes et aux hydrocarbures chlorés (tétrachloroéthylène) (30). Les larves filariformes peuvent aussi être inactivées par de l'eau chauffée à plus de $80{ }^{\circ} \mathrm{C}(30)$.

Les contacts familiaux des patients atteints de strongyloïdose disséminée ou d'un syndrome d'hyperinfection par Strongyloides doivent faire l'objet d'un dépistage sérologique de la strongyloïdose et d'un examen des selles afin d'identifier une transmission interhumaine.

\section{Algorithme de diagnostic et de traitement pour la strongyloïdose - Étapes 1 à 4}

Note à l'intention du lecteur : toutes les étapes doivent être exécutées dans l'ordre, car l'étape 3 nécessite les données des étapes 1 et 2.

Étape 1 : Définir la catégorie de risque pour la strongyloïdose disséminée sur la base des facteurs épidémiologiques et cliniques

\begin{tabular}{|c|c|c|}
\hline \multirow[b]{2}{*}{$\begin{array}{l}\text { Catégorie de risque épidémiologique pour } \\
\text { l'exposition au Strongyloides ou l'infection } \\
\text { par Strongyloides }\end{array}$} & \multicolumn{2}{|c|}{ Facteur de risque clinique de strongyloïdose disséminée } \\
\hline & $\begin{array}{l}\text { - Infection par le HTLV-1 }{ }^{1} \\
\text { - Traitement aux glucocorticoïdes }{ }^{2} \\
\text { - Agent immunomodulateur } \\
\text { - Hématopathie maligne }\end{array}$ & $\begin{array}{l}\text { - Aucune anomalie connue dans } \\
\text { I'immunité à médiation cellulaire }\end{array}$ \\
\hline $\begin{array}{l}\text { Naissance, résidence ou séjour de longue durée }{ }^{4} \text { en Asie } \\
\text { du Sud-Est, en Océanie, en Afrique subsaharienne, en } \\
\text { Amérique du Sud, dans les Caraïbes }\end{array}$ & Élevé & Modéré \\
\hline $\begin{array}{l}\text { Naissance, résidence ou séjour de longue durée }{ }^{4} \text { dans les } \\
\text { pays méditerranéens, au Moyen-Orient, en Afrique du } \\
\text { Nord, dans le sous-continent indien, en Asie }\end{array}$ & Modéré & Faible \\
\hline $\begin{array}{l}\text { Naissance, résidence ou séjour de longue durée }{ }^{4} \text { en } \\
\text { Australie, en Amérique du Nord ou en Europe de } \\
\text { l'Ouest5 }\end{array}$ & Très faible & Très faible \\
\hline \multicolumn{3}{|l|}{ HTLV-1 = virus T-lymphotrope humain intercurrent } \\
\hline \multicolumn{3}{|c|}{$\begin{array}{l}\text { Agents inclus : les agents alcoylants, les antimétabolites, les agents immunosuppresseurs ou immunomodulateurs utilisés dans la prise en charge des transplantations d'organe plein et la sclérose en } \\
\text { plaques, les agents de blocage du facteur de nécrose tumorale, les agents anti-IL-1, les agents bloquant l'adhésion et les agents provoquant la diminution du nombre de lymphocytes. }\end{array}$} \\
\hline \multicolumn{3}{|c|}{$\begin{array}{l}\text { Exposition cumulée de six mois dans des zones rurales ou des plages, ou contact de la peau avec le sable ou la terre dans une zone à risque, y compris dans le cadre d'un voyage de plus courte } \\
\text { durée }(8,9,10) \text {. En cas de réexposition significative prolongée, envisagez de procéder à un nouveau dépistage si le test était initialement négatif. }\end{array}$} \\
\hline \multicolumn{3}{|c|}{$\begin{array}{l}\text { Les régions d'Amérique du Nord présentant un risque supérieur à un faible risque incluent la Floride, le Kentucky et la Virginie. Les Aborigènes australiens présentent un risque élevé de } \\
\text { strongyloïdose. }\end{array}$} \\
\hline
\end{tabular}




\section{Étape 2 : Définir le syndrome clinique suspect}

\begin{tabular}{|c|c|c|}
\hline Syndrome clinique suspecté & Test diagnostique approprié & $\begin{array}{l}\text { Prélèvement } \\
\text { diagnostique approprié }\end{array}$ \\
\hline $\begin{array}{l}\text { Éosinophilie } \pm \text { asymptomatique (les } \\
\text { personnes asymptomatiques subissant } \\
\text { une immunosuppression prévue peuvent } \\
\text { être incluses) } \\
\text { (Risque très faible) }\end{array}$ & $\begin{array}{l}\text { - Sérologie } \\
\text { - Examen des selles (œufs et parasites) }\end{array}$ & $\begin{array}{l}\text { - Sérum } \\
\text { - Échantillon de selles fixées au SAF }\end{array}$ \\
\hline $\begin{array}{l}\text { Strongyloïdose intestinale simple }{ }^{1} \\
\text { (Risque faible) }\end{array}$ & $\begin{array}{l}\text { - Sérologie } \\
\text { - Examen des selles (œufs et parasites) }\end{array}$ & $\begin{array}{l}\text { - Sérum } \\
\text { - Échantillon de selles fixées au SAF }\end{array}$ \\
\hline $\begin{array}{l}\text { Syndrome d'hyperinfection peu sévère }{ }^{2} \\
\text { (Risque modéré) }\end{array}$ & $\begin{array}{l}\text { - Sérologie } \\
\text { - Examen des selles (œufs et parasites) } \\
\text { - Examen des expectorations (œufs et parasites) } \\
\text { - Culture sur plaque }\end{array}$ & $\begin{array}{l}\text { - Sérum } \\
\text { - Échantillon de selles fixées au SAF } \\
\text { - Expectoration fraîche dans un contenant } \\
\text { stérile } \\
\text { - Selles fraîches/expectoration fraîche pour } \\
\text { culture sur plaque }\end{array}$ \\
\hline $\begin{array}{l}\text { Strongyloïdose disséminée }{ }^{3} \\
\text { (Risque élevé) }\end{array}$ & $\begin{array}{l}\text { - Sérologie } \\
\text { - Examen des selles (œufs et parasites) } \\
\text { - Examen des expectorations (œufs et parasites) } \\
\text { - Analyse d'urine (œufs et parasites) } \\
\text { - Analyse du liquide céphalorachidien (œufs et } \\
\text { - } \text { - Exarasites) } \\
\text { - Culture des tissus (œufs et parasites) }\end{array}$ & $\begin{array}{l}\text { - Sérum } \\
\text { - Échantillon de selles fixées au SAF } \\
\text { - Expectoration fraîche dans un contenant } \\
\text { stérile } \\
\text { - Urine dans un contenant stérile } \\
\text { - Liquide céphalorachidien dans un contenant } \\
\text { stérile } \\
\text { - Tissu, inclus dans la paraffine ou non traité } \\
\text { - Tout nouvel échantillon, tel qu'il est décrit ci- } \\
\text { dessus, pour culture sur plaque }\end{array}$ \\
\hline
\end{tabular}

1 Caractérisée par une perte de poids, un malaise abdominale et des selles molles, avec ou sans éosinophilie.

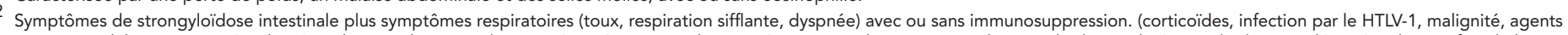

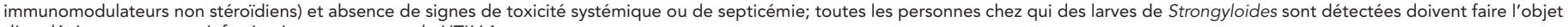
d'un dépistage pour une infection intercurrente par le HTLV-1.

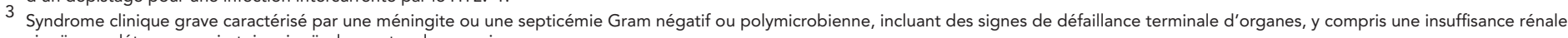
aiguë, une détresse respiratoire aiguë, des pertes de connaissance, un coma.

$4 \mathrm{SAF}=$ Sodium acetate-acetic acid-formalin (en anglais) 
Étape 3 : Approche suggérée pour le diagnostic et la prise en charge empirique, en fonction de la catégorie de risque identifiée (Étape 1) et du syndrome clinique (Étape 2)

\begin{tabular}{|c|c|c|c|c|}
\hline \multirow[b]{2}{*}{$\begin{array}{l}\text { Catégorie de risque } \\
\text { (conformément à } \\
\text { l'étape 1) }\end{array}$} & \multicolumn{4}{|c|}{ Syndrome clinique suspecté (conformément à l'étape 2) } \\
\hline & $\begin{array}{l}\text { Éosinophilie } \pm \\
\text { asymptomatique }^{1}\end{array}$ & $\begin{array}{l}\text { Strongyloïdose } \\
\text { intestinale simple }\end{array}$ & $\begin{array}{l}\text { Syndrome } \\
\text { d'hyperinfection peu } \\
\text { sévère }\end{array}$ & $\begin{array}{l}\text { Strongyloïdose } \\
\text { disséminée }\end{array}$ \\
\hline Élevé & $\begin{array}{l}\text { Envoyer les échantillons } \\
\text { appropriés à des fins de } \\
\text { dépistage }^{2} \\
\text { (Risque modéré) }\end{array}$ & $\begin{array}{l}\text { Traitement empirique dans } \\
\text { I'attente du dépistage } \\
\text { (Risque élevé) }\end{array}$ & $\begin{array}{l}\text { Traitement empirique dans } \\
\text { I'attente du dépistage } \\
\text { (Risque élevé) }\end{array}$ & $\begin{array}{l}\text { Traitement empirique dans } \\
\text { I'attente du dépistage } \\
\text { (Risque élevé) }\end{array}$ \\
\hline Modéré & $\begin{array}{l}\text { Envoyer les échantillons } \\
\text { appropriés à des fins de } \\
\text { dépistage } \\
\text { (Risque modéré) }\end{array}$ & $\begin{array}{l}\text { Envoyer les échantillons } \\
\text { appropriés à des fins de } \\
\text { dépistage } \\
\text { (Risque modéré) }\end{array}$ & $\begin{array}{l}\text { Traitement empirique dans } \\
\text { I'attente du dépistage } \\
\text { (Risque élevé) }\end{array}$ & $\begin{array}{l}\text { Traitement empirique dans } \\
\text { I'attente du dépistage } \\
\text { (Risque élevé) }\end{array}$ \\
\hline Faible & $\begin{array}{l}\text { Envoyer les échantillons } \\
\text { appropriés à des fins de } \\
\text { dépistage } \\
\text { (Risque faible) }\end{array}$ & $\begin{array}{l}\text { Envoyer les échantillons } \\
\text { appropriés à des fins de } \\
\text { dépistage } \\
\text { (Risque faible) }\end{array}$ & $\begin{array}{l}\text { Envoyer les échantillons } \\
\text { appropriés à des fins de } \\
\text { dépistage } \\
\text { (Risque faible) }\end{array}$ & $\begin{array}{l}\text { Envoyer les échantillons } \\
\text { appropriés à des fins de } \\
\text { dépistage } \\
\text { (Risque faible) }\end{array}$ \\
\hline Très faible & $\begin{array}{l}\text { Dépistage non } \\
\text { recommandé. Envisager un } \\
\text { autre diagnostic } \\
\text { (Risque très faible) }\end{array}$ & $\begin{array}{l}\text { Dépistage non } \\
\text { recommandé. Envisager un } \\
\text { autre diagnostic } \\
\text { (Risque très faible) }\end{array}$ & $\begin{array}{l}\text { Envoyer les échantillons } \\
\text { appropriés à des fins de } \\
\text { dépistage } \\
\text { (Risque très faible) }\end{array}$ & $\begin{array}{l}\text { Envoyer les échantillons } \\
\text { appropriés à des fins de } \\
\text { dépistage } \\
\text { (Risque très faible) }\end{array}$ \\
\hline
\end{tabular}

1 Ceci inclut les personnes asymptomatiques subissant une immunosuppression prévue.

2 Dans les rares cas où le patient est considéré comme présentant un risque élevé de strongyloïdose et d'immunosuppression, et ne peut pas attendre le test diagnostique définitif, nous recommandons un traitement empirique de deux doses d'ivermectine, comme il est indiqué dans l'étape 4 ci-dessous. 


\section{Étape 4 : Traiter la strongyloïdose en fonction du syndrome clinique et des résultats des tests diagnostiques}

\begin{tabular}{|c|c|c|c|}
\hline Syndrome clinique & Diagnostic confirmé par & Gestion des adultes & Gestion pédiatrique \\
\hline $\begin{array}{l}\text { Éosinophilie } \pm \\
\text { asymptomatique (y } \\
\text { compris les personnes } \\
\text { asymtomatiques subissant } \\
\text { une immunosuppression } \\
\text { prévue) } \\
\text { (Risque très faible) }\end{array}$ & $\begin{array}{l}\text { - Sérologie } \\
\text { - Examen des selles (œufs et } \\
\text { parasites) pour la recherche de } \\
\text { larves }\end{array}$ & $\begin{array}{l}\text { Ivermectine } 200 \mu \mathrm{g} / \mathrm{kg} \text { par jour p.o. } \\
\text { une fois par jour x } 2 \text { doses le jour } 1 \\
\text { et } 2 \text {, ou à } 14 \text { jours d'intervalle }{ }^{1}\end{array}$ & $\begin{array}{l}\text { Ivermectine } 200 \mu \mathrm{g} / \mathrm{kg} \text { par jour p.o. } \\
\text { une fois par jour x } 2 \text { doses le jour } 1 \\
\text { et } 2 \text {, ou à } 14 \text { jours d'intervalle }{ }^{1}\end{array}$ \\
\hline $\begin{array}{l}\text { Strongyloïdose intestinale } \\
\text { simple }^{2} \text {, } \\
\text { (Risque faible) }\end{array}$ & $\begin{array}{l}\text { - Sérologie } \\
\text { - Examen des selles (œufs et } \\
\text { parasites) pour la recherche de } \\
\text { larves }\end{array}$ & $\begin{array}{l}\text { Ivermectine } 200 \mu \mathrm{g} / \mathrm{kg} \text { par jour p.o. } \\
\text { une fois par jour x } 2 \text { doses le jour } 1 \\
\text { et } 2 \text {, ou à } 14 \text { jours d'intervalle }{ }^{1}\end{array}$ & $\begin{array}{l}\text { Ivermectine } 200 \mu \mathrm{g} / \mathrm{kg} \text { par jour p.o. } \\
\text { une fois par jour x } 2 \text { doses le jour } 1 \\
\text { et } 2 \text {, ou à } 14 \text { jours d'intervalle }{ }^{1}\end{array}$ \\
\hline $\begin{array}{l}\text { Syndrome d'hyperinfection } \\
\text { peu sévère } \\
\text { (Risque modéré) }\end{array}$ & $\begin{array}{l}\text { - Sérologie } \\
\text { - Examen des selles (œufs et } \\
\text { parasites) pour la recherche de } \\
\text { larves } \\
\text { - Examen des expectorations } \\
\text { (œufs et parasites) pour la } \\
\text { recherche de larves }\end{array}$ & $\begin{array}{l}\text { Ivermectine } 200 \mu \mathrm{g} / \mathrm{kg} \text { par jour p.o. } \\
\text { une fois par jour } \times 2 \text { doses le jour } 1 \\
\text { et } 2 \text {, ou à } 14 \text { jours d'intervalle }{ }^{1} \\
\text { PLUS } \\
\text { Albendazole, } 400 \mathrm{mg}, \text { p.o. } 2 \text { f.p.j. } \\
\text { pendant } 7 \text { jours } \\
\text { OU, en monothérapie: } \\
\text { Ivermectine } 200 \mu \mathrm{g} / \mathrm{kg} \text { par jour p.o. } \\
\text { une fois par jour pendant } 7 \text { jours }\end{array}$ & $\begin{array}{l}\text { Ivermectine } 200 \mu \mathrm{g} / \mathrm{kg} \text { par jour p.o. } \\
\text { une fois par jour } \times 2 \text { doses le jour } 1 \\
\text { et } 2 \text {, ou à } 14 \text { jours d'intervalle }{ }^{1} \\
\text { PLUS } \\
\text { Albendazole, } 400 \mathrm{mg} \text {, p.o. } 2 \text { f.p.j. } \\
\text { pendant } 7 \text { jours } \\
\text { OU, en monothérapie : } \\
\text { Ivermectine } 200 \mu \mathrm{g} / \mathrm{kg} \text { par jour p.o. } \\
\text { une fois par jour pendant } 7 \text { jours }\end{array}$ \\
\hline $\begin{array}{l}\text { Strongyloïdose disséminée }{ }^{4,5} \\
\text { (Risque élevé) }\end{array}$ & $\begin{array}{l}\text { - Sérologie } \\
\text { - Examen des selles (œufs et } \\
\text { parasites) pour la recherche de } \\
\text { larves } \\
\text { - Examen des expectorations } \\
\text { (œufs et parasites) pour la } \\
\text { recherche de larves- Analyse } \\
\text { d'urine, examen de liquide } \\
\text { céphalorachidien ou d'autres } \\
\text { tissus ou liquides corporels pour } \\
\text { la recherche de larves }\end{array}$ & $\begin{array}{l}\text { Ivermectine } 200 \mu \mathrm{g} / \mathrm{kg} \text { par jour p.o. } \\
\text { ou par voie sous cutanée }{ }^{6} \text { une fois } \\
\text { par jour } \\
\text { PLUS } \\
\text { Albendazole } 400 \mathrm{mg} \text { p.o. } \\
2 \text { f.p.j. jusqu'à cessation du } \\
\text { développement larvaire et } \\
\text { amélioration clinique }\end{array}$ & $\begin{array}{l}\text { Ivermectine } 200 \mu \mathrm{g} / \mathrm{kg} \text { par jour p.o. } \\
\text { ou par voie sous cutanée }{ }^{6} \text { une fois } \\
\text { par jour } \\
\text { PLUS } \\
\text { Albendazole } 400 \mathrm{mg} \text { p.o. } \\
2 \text { f.p.j. jusqu'à cessation du } \\
\text { développement larvaire et } \\
\text { amélioration clinique }\end{array}$ \\
\hline
\end{tabular}

1 Un intervalle de 14 jours entre les doses est préférable en raison du risque d'infection pré-symptomatique découlant d'une auto-infection (15).

2 Caractérisée par une perte de poids, une gêne abdominale et des selles molles, avec ou sans éosinophilie.

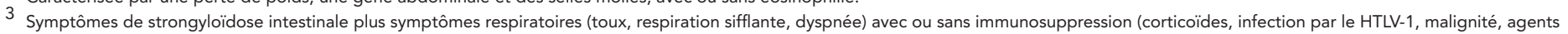

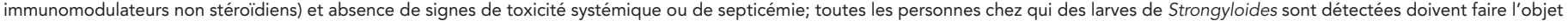
d'un dépistage pour une infection intercurrente par le HTLV-1.

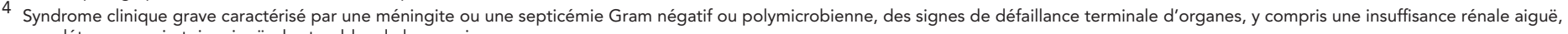
une détresse respiratoire aiguë, des troubles de la conscience, un coma.

5 Les patients atteints de strongylö̈dose disséminée doivent également recevoir un traitement empirique pour la septicémie polymicrobienne avec des antibiotiques à spectre large.

6 Disponible uniquement en formulation vétérinaire; I'utilisation chez l'homme n'est pas indiquée sur l'étiquette et n'est pas approuvée par Santé Canada (25,31,32,33). 


\section{Conclusion}

La strongyloïdose est relativement répandue au sein de la population migrante mondiale. Le dépistage de la maladie doit s'appuyer sur une évaluation des risques individuels, en tenant compte du risque d'exposition au Strongyloides, du risque de maladie disséminée et de la présence d'un syndrome clinique (ce qui peut inclure les personnes asymptomatiques qui doivent subir une immunosuppression iatrogène). La présente déclaration résume les renseignements pertinents disponibles sur la strongyloïdose et fournit un outil pratique destiné aux cliniciens pour la prévention, l'évaluation et la prise en charge de la strongyloïdose disséminée au Canada.

\section{Points clés}

- Le dépistage de la strongyloïdose doit être envisagé pour les personnes sujettes à un risque épidémiologique ou de comorbidités qui les exposent à un risque d'hyperinfection et de dissémination du Strongyloides. Les personnes exposées au risque le plus élevé d'hyperinfection et de dissémination sont celles qui sont nées dans une région où le Strongyloides est endémique, qui présentent une immunodépression iatrogène ou une infection par le virus HTLV-1 intercurrent.

- Le diagnostic de la strongyloïdose est fondé sur des tests sérologiques ou l'examen des selles et autres échantillons cliniques pour la recherche de larves. La sérologie est généralement très sensible, tandis que l'examen des selles est très spécifique.

- Les caractéristiques de performance des tests diagnostiques peuvent être altérées par l'immunosuppression et les co-infections comme le HTLV-1. La sensibilité de l'examen des selles peut augmenter, tandis que la sensibilité de la sérologie peut diminuer.

- II est recommandé de faire appel à un spécialiste en médecine tropicale ayant une expertise dans la prise en charge de la strongyloïdose pour tout patient ayant une strongyloïdose suspectée ou confirmée et pour les patients souffrant d'une infection par Strongyloides et par le HTLV-1 ou Loa loa.

\section{Remerciements}

Le CCMTMV est reconnaissant de la contribution de Mihaela Gheorghe.

Membres du CCMTMV : McCarthy A (présidente), Boggild A, Brophy J, Bui Y, Crockett M, Greenaway C, Libman M, Teitelbaum $P$ et Vaughan $\mathrm{S}$.

Membres de liaison : Audcent T (Société canadienne de pédiatrie), Gershman M (Centers for Disease Control and Prevention des États-Unis) et Pernica J (Association pour la microbiologie médicale et l'infectiologie Canada).

Membres d'office : Marion D (Centre des services de santé des Forces canadiennes, ministère de la Défense nationale),
McDonald P (Division des médicaments anti-infectieux, Santé Canada), Schofield S (Entomologie de la lutte antiparasitaire, ministère de la Défense nationale) et Tepper M (Direction de la protection de la santé de la Force, ministère de la Défense nationale).

\section{Conflit d'intérêts}

Aucun.

\section{Financement}

Ce travail a été appuyé par l'Agence de la santé publique du Canada.

\section{Références}

1. World Health Organization. Intestinal Worms: Strongyloidiasis. Geneva: WHO; 2015. http://www.who.int/ intestinal_worms/epidemiology/strongyloidiasis/en.

2. Centers for Disease Control and Prevention. Parasites Strongyloides. Epidemiology and risk factors. Atlanta GA: CDC; 2015. http://www.cdc. gov/parasites/strongyloides/ epi.html.

3. Schär F, Trostdorf U, Giardina F, Khieu V, Muth S, Marti H, et al. Strongyloides stercoralis: Global distribution and risk factors. PLoS Negl Trop Dis. 2013;7(7):e2288.

4. Gyorkos TW, Genta RM, Viens P, MacLean JD. Seroepidemiology of Strongyloides infection in the Southeast Asian refugee population in Canada. Am J Epidemiol. 1990;132:257-64.

5. Boggild AK, Geduld J, Libman M, McCarthy A, Vincelette J, Ghesquiere W, Hajek J, Kuhn S, Freedman DO, Kain KC. Travel-acquired infections in Canada: CanTravNet 2011 2012. CCDR. 2014;40(16):313-325.

(Disponible en français : http://www.phac-aspc.gc.ca/ publicat/ccdr-rmtc/14vol40/dr-rm40-16/dr-rm40-16-surv-fra. php).

6. Boggild AK, Geduld J, Libman M, Ward B, McCarthy A, Doyle P, Ghesquiere W, Vincelette J, Kuhn S, Freedman DO, Kain KC. Travel-acquired infections and illnesses in Canadians: Surveillance report from CanTravNet surveillance data, 2009 - 2011. Open Med. 2014;8(1):e20-e32.

7. Statistics Canada. Immigration and ethnocultural diversity in Canada. National Household Survey, 2011. Ottawa ON: Minister of Industry; 2015. (Disponible en français : http:// www12.statcan.gc.ca/nhs-enm/2011/as-sa/99-010-x/99-010x2011001-fra.pdf).

8. Baaten GG, Sonder GJ, van Gool T, et al. Travel-related schistosomiasis, strongyloidiasis, filariasis, and toxocariasis: The risk of infection and the diagnostic relevance of blood eosinophilia. BMC Infect Dis. 2011;11:84.

9. Angheben A, Mistretta M, Gobbo M, Bonafini S, lacovazzi T, Sepe A, Gobbi F, Marocco S, Rossanese A, Bisoffi Z. Acute strongyloidiasis in Italian tourists returning from Southeast Asia. J Travel Med. 2011 Mar-Apr;18(2):138-40. 
10. Bailey KE, Danylo A, Boggild AK. Chronic larva currens following tourist travel to the Gambia and Southeast Asia over 20 years ago. J Cutan Med Surg. 2015;19(4):412-5.

11. Lim S, Katz K, Kradjen S, Fuksa M, Keystone JS, Kain KC. Complicated and fatal Strongyloides infection in Canadians: Risk factors, diagnosis, and management. CMAJ. 2004;171(5):479-84.

12. Rogers WA Jr, Nelson B. Strongyloidiasis and malignant lymphoma "opportunistic infection" by a nematode. JAMA. 1966;195:685-7.

13. Willis AJP, Nwokolo C. Steroid therapy and strongyloidiasis. Lancet. 1966;1:1396-8.

14. Cruz R, Reboucas G, Rocha H. Fatal strongyloidiasis in patients receiving corticosteroids. N Engl J Med. 1966;275:1093-6.

15. Suputtamongkol $Y$, Premasathian N, Bhumimuang $K$, Waywa D, Nilganuwong S, Karuphong E, et al. Efficacy and safety of single and double doses of ivermectin versus seven-day high dose albendazole for chronic strongyloidiasis. PLoS Negl Trop Dis. 2011;5(5):e1044.

16. Igra-Siegman $Y$, Kapila R, Sen P, Kaminski ZC, Louria DB. Syndrome of hyperinfection with Strongyloides stercoralis. Rev Infect Dis. 1981;3:397-407.

17. Pottie K, Greenaway C, Feightner J, Welch V, Swinkels $\mathrm{H}$, Rashid $\mathrm{M}$, et al. Evidence-based clinical guidelines for immigrants and refugees. CMAJ Can Med Assoc J. 2011;183(12):E824-E925.

18. Porto AF, Oliveira Filho J, Neva FA, Orge G, Alcântara L, Gam A, Carvalho EM. Influence of human T-cell lymphocytotropic virus type 1 infection on serologic and skin tests for strongyloidiasis. Am J Trop Med Hyg. 2001 Nov;65(5):610-3.

19. Schaffel R, Nucci M, Carvalho E, Braga M, Almeida L, Portugal R, Pulcheri W. The value of an immunoenzymatic test (enzyme-linked immunosorbent assay) for the diagnosis of strongyloidiasis in patients immunosuppressed by hematologic malignancies. Am J Trop Med Hyg. 2001 Oct;65(4):346-50.

20. Public Health Agency of Canada. Canadian Malaria Network (CMN). (Disponible en français: http://www.phac-aspc.gc.ca/ tmp-pmv/quinine/index-fra.php).

21. Meunnig P, Pallin D, Challah C, Khan K. The costeffectiveness of ivermectin vs. albendazole in the presumptive treatment of strongyloidiasis in immigrants to the United States. Epidemiol Infect. 2004;132:1055-1063.

22. Balagopal A, Mills L, Shah A, Subramanian A. Detection and treatment of Strongyloides hyperinfection syndrome following lung transplantation. Transpl Infect Dis. 2009;11:149-154.

23. Altintop L, Cakar B, Hokelek M, Bektas A, Yildiz L, Karaoglanoglu M. Strongyloides stercoralis hyperinfection in a patient with rheumatoid arthritis and bronchial asthma: A case report. Ann Clin Microbiol Antimicrob. 2010;9:27.

24. Hasan A, Le M, Pasko J, Ravin KA, Clauss H, Hasz R, et al. Transmission of Strongyloides stercoralis through transplantation of solid organs - Pennsylvania, 2012. MMWR Morbid Mortal Wkly Rep. 2013;62(14):264-266.
25. Chiodini PL, Reid AJC, Wiselka MJ, Firmin R, Foweraker J. Parenteral ivermectin in Strongyloides hyperinfection. Lancet. 2000;355:43-44.

26. Roxby AC, Gottlieb GS, Limaye AP. Strongyloidiasis in transplant patients. Clin Infect Dis. 2009;49:1411-1423.

27. Health Canada. Guidance document for industry and practitioners: Special access programme for drugs. Ottawa ON: Health Canada; 2013. (Disponible en français : http:// www.hc-sc.gc.ca/dhp-mps/alt_formats/hpfb-dgpsa/pdf/ acces/sapg3_pasg3-fra.pdf).

28. Maraha B, Buiting AGM, Hol C, Pelgrom R, Blotkamp C, Polderman AM. The risk of Strongyloides stercoralis transmission from patients with disseminated strongyloidiasis to the medical staff. J Hosp Infect. 2001;49:222-224.

29. Hauber HP, Galle J, Chiodini PL, Rupp J, Birke R, Vollmer E, Zabel P, Lange C. Fatal outcome of a hyperinfection syndrome despite successful eradication of Strongyloides with subcutaneous ivermectin. Infect. 2005;33(5/6):383-386.

30. Public Health Agency of Canada. Ancylostoma duodenale. Pathogen safety data sheet - infectious substances. Ottawa ON: PHAC; 2012. (Disponible en français : http://www.phacaspc.gc.ca/lab-bio/res/psds-ftss/ancylostoma-duodenalefra).

31. Turner SA, Maclean JD, Fleckenstein L, Greenaway C. Parenteral administration of ivermectin in a patient with disseminated strongyloidiasis. Am J Trop Med Hyg. 2005;73(5):911-914.

32. Leung V, Al-Rawahi GN, Grant J, Fleckenstein L, Bowie W. Case report: Failure of subcutaneous ivermectin in treating Strongyloides hyperinfection. Am J Trop Med Hyg. 2008;79(6):853-855.

33. Salluh JIF, Feres GA, Velasco E, Holanda GS, Toscano $L$, Soares M. Successful use of parenteral ivermectin in an immunosuppressed patient with disseminated strongyloidiasis and septic shock. Intensive Care Med. 2005;31:1292. 